\title{
Exploitation of Semantic Web Technology in ERP Systems
}

\author{
Amin Anjomshoaa, Shuaib Karim, Ferial Shayeganfar, and A Min Tjoa \\ Institute of software technology and Interactive Systems \\ Vienna University of Technology \\ Favoritenstrasse 9-11, 1040 Vienna, Austria \\ \{andjomshoaa, skarim, ferial, amin\}@ifs.tuwien.ac.at \\ WWW home page: http://www.ifs.tuwien.ac.at
}

\begin{abstract}
Until recently existing technologies enabling the integration of enterprise systems, use very few of the capabilities of modern research. For example, the activity of finding services, which should deliver expected enterprise functionality, has to be driven by humans. The process of assembling pieces of functionality into complex business processes also involves human interaction. The semantic web technology can be used in the context of an ERP to enable the lacking automation process. This technology can significantly contribute to overcome the deficiencies rooting from the heterogeneity of information contents and semantics generated from various sources. A challenge in Enterprise Information System is the detailed capture of activities and process information. The semantic integration of Personal Information Management Systems could be regarded as a first step towards the modernization of ERP applications. We also describe the opportunities of significantly improving business security by using ontologies. The use of ontology concepts of the Semantic Web can be regarded as an essential step in the evolution of Service Oriented Architectures for ERP applications.
\end{abstract}

\section{Introduction}

Enterprise Resource Planning (ERP) is a business management system that integrates all facets of our business including marketing, finance, inventory control, material ordering, production planning, manufacturing, testing, sales, delivery, and after sales customer support. Today the ERP systems have become modular and configurable to suit our needs. In ERP environment software applications perform

Please use the following format when citing this chapter:

Anjomshoaa, A., Karim, S., Shayeganfar, F., Tjoa, A., Min, 2006, in International Federation for Information Processing, Volume 205, Rescarch and Practical Issues of Enterprisc Information Systems, eds. Tjoa, A.M., Xu, L., Chaudhry, S., (Boston:Springer), pp.417-427. 
business tasks in a modular way depending upon the choice and nature of our business. Practically these modules are concerned with separate domains and are guided by human experts in those domains. The ERP is supposed to work for the achievement of common goals of the company. Different modules require different vocabulary and concept hierarchy. Nevertheless they must interact with each other for transforming the customer order into profitability.

Some of the major investments a company need to do even after deployment of ERP solution is to invest a lot on software customization, training of personnel, integration and testing of different modules, data conversion and analysis. Yet after considerable post-deployment time the profitability seems far away. The main responsible factors are the heterogeneity of information contents and the semantics generated from various sources like customer requirements, vendors, regulation bodies, and company's internal departments (mentioned at the beginning of Introduction section). These problems can be significantly addressed by exploiting semantic web technology in ERP systems.

The Semantic Web is a web of data that will enable machines to comprehend the data. Until recently the information available on World Wide Web is solely human understandable. The goal of Semantic Web is to change this information into Knowledge resources. In the Semantic Web the information will have a well defined meaning and this will enable the computers to know how to process the information. Next generation of World Wide Web will be greatly affected by this new evolving technology and it is clear that this will have tremendous economical impacts. It is important to note that the semantic web technology can also be used in a localized context such as ERP for our organizations, yet giving an edge to be in a state of readiness for integrating and sharing our business as specific web services to outside world.

In Semantic Web the information model is represented as a graph with nodes representing the resources using unique identifiers called Uniform Resource Identifier (URI) [1], and the edges representing the relationship between them with the help of properties for a specific domain of discourse. Resource can be anything such as a person, a document, a product technical drawing, a testing tool specification, a business process or a service which is further described at the specified address pointed by the URI. The representation between the nodes and the edges is formally specified in a machine processable way which can also be shared globally by virtue of its URI and an agreed upon schema. The formal information representation is made using Resource Description Framework (RDF) [2] which defines an XML syntax for RDF in form of Subject-Predicate-Object triples. The richness of the knowledge base is further enhanced by describing inference rules with the help of RDF Semantics (RDFS) [3] and making it sharable by using Web Ontology Language (OWL) [4]. The information thus represented is a "specification of a conceptualization" or ontology [5]. In simple terms it is a set of shared vocabulary, arrangement of related taxonomy, and the definition of axioms to specify the relationships between them. Once the triples are expressed in RDF, then these can be accessed programmatically by the software agents designed to carry out certain services over the web. The service description about how it runs, as well as 
the explicit logic to describe consequences of using the service are also made machine-readable [6]. The theoretical flexibility to make shareable ontologies on a global scale poses many challenges in merging, integration and versioning of ontologies that are not completely solved so far. However, by making use of suitable ontological commitments the ontologies can successfully be made sharable in the concerned domain such as an organizational ERP system. Ontological commitments are agreements to use the shared vocabulary in a coherent and consistent manner [5].

\section{State of the Art and Current Works}

There are an increasing number of efforts to integrate Semantic Web based solutions in business. Especially there have been many advances in Business to Business (B2B) integration via Semantic Web Services. To quote an example the Digital Enterprise Research Institute (DERI) tries to address intelligent Web Services upon Semantic Web technologies [7]. Unlike the current web service technologies which provide limited support in mechanizing service recognition, service configuration and combination (i.e., realizing complex workflows and business logics with Web services), DERI focuses on service comparison and automated negotiation of services. In a business environment, the vision of flexible and autonomous Web service translates into automatic cooperation between enterprise services. Any enterprise requiring a business interaction with another enterprise can automatically discover and select the appropriate optimal Web services relying on selection policies. Services can be invoked automatically and payment processes can be initiated. Any necessary mediation would be applied based on data and process ontologies and the automatic translation and semantic interoperation.

Another yet more important activity is creating upper ontologies for the problem domain. ERPs are an example where a lack of common comprehensible standards and ontology has contributed to massive failures [8]. Since technical terms are often unclear, and not described in the language of business, many inter-organizational negotiations are ambiguous. Therefore, without an upper ontology the global communication between businesses is not possible. One such model which was originally designed for accounting is Resource-Event-Agent (REA) business model. Later REA business model was extended for supply chain management [9]. The key extension was the Dependent Demand relationship as defined by Manufacturing Resource Planning (MRP) systems [9]. As a semantic Web, the REA model makes it possible to link economic events together across different companies, industries, and nations. The semantic types of these links are activity-to-activity or agent-to-agent or person-to-person, not just company-to-company. This means that each individual in a REA based supply chain can be linked directly to every other individual.

SCOR+ is another more specific ontology for the description of business processes which has been developed based on Supply Chain Operations Reference model (SCOR model). The SCOR model is a process reference model that has been developed and endorsed by the Supply-Chain Council as the cross-industry standard 
diagnostic tool for supply-chain management. SCOR enables users to address, improve, and communicate supply-chain management practices within and between all interested parties [10]. SCOR model can define the supply chains, measure its performance, and benchmark it against industry standards. This model defines the supply chain in terms of processes, performance metrics, and best practices. It has already been used and extended to develop an ontology for supply chain simulation modeling [11]. SCOR+ is directed towards overcoming the limitations of the basic SCOR model through an easy to use ontology based tool that enables an automated and comprehensive definition of the Supply Chain at any level of detail. SCOR+ captures all the constituent parts of the Supply Chain at four different levels which are, the supply chain level, the enterprise level, the element level, and the interaction level [12].

\section{Business Opportunities with Semantic Web}

The most obvious application of Semantic Web in business field is changing the web to a processable resource for machines. In this section we will inspect the possible uses of Semantic Web technology in the fields of Business and Economics. The information which is available on Web today is mostly produced to be humanunderstandable. The process of changing this information to Knowledge is time and budget consuming. On the other hand the policy makers need to have access to the last updated information and usually the human-based method of information processing is not economical.

Living systems have different characteristics like self-regulation of processes, reproduction and growth [13]. Nevertheless, these relevant characteristics could be envisioned in a semantic way in business knowledge management. Ontologies of business items grow and reproduce new ones with processes and services. These ontologies include information about our business objects such as business partners, suppliers, customers, persons, places, organizations, events and business processes. This could be of great importance in office systems including Customer Relationship Management (CRM), e-business and Supplier Relationship Management (SRM).

In the physical world, entities are usually interconnected, either by physical or by semantic means. In the latter case, the semantic meaning is added by human interaction (in an abstract sense) with the physical world. Business items in our case can be understood as information entities (in some cases they are representations of such physical entities) stored according to ontologies in a semantic database, which are connected to other information entities according to their semantic meaning. Also ontologies 'live' in a way, as they develop and modify permanently during the system and business lifetime.

Current Web technologies are highly efficient in processing data for human reception, i.e., the transformation from data to information. However the 'generation of meaning' is up to the human. A great deal of effort has already been made, and work is still going on to represent semantics explicitly on the Web. This is required 
to give computer systems the capability to enhance preprocessing of huge amounts of data for the user. It becomes more important as the 'awareness radius' of the contemporary knowledge worker and consumer is continuously increasing. This, results from the observation, that users do not limit their information search to specific data repositories, like searching for an address or an event in a calendar any longer. The availability of databases under common or similar interfaces (like webpages) creates the demand to express more complex queries demanding information aggregated from many different systems using different semantic concepts.

\subsection{ERP Implementation}

Business analysis and Business reengineering is embedded in a complex web of relationships that determines processes and methods in ERP implementation. An ERP implementation process relies on large databases and their interrelations that are often managed by human knowledge and interactions. Some of these relationships are strongly coupled to and affected from contextual parameters like country-specific policies. Migration of existing context attributes and applying them to business entities is a big challenge. Another important issue is keeping track of changes and adopting the business processes, which is not easy with the existing ERP solutions.

During an ERP implementation phase the business engineer should make some critical decisions about business objects and methods to be used. Also the business criteria and requirements should be considered and checked at all phases of business evolution. These represent a heterogeneous, dynamic, and frequently ill-structured set of informational resources, thus frequently exacerbating the efforts for designers and planners to identify, obtain, and process information in an effective and timely manner.

In the real world the product lines usually require high technological and maintenance intensive assets. In addition the experience of employees and their know-how is the most critical success factor. Regarding these facts, the changing of company's strategy and product portfolio is a long term issue that must be planned after intensive marketing and feasibility studies. Management and analysis of such huge amount of data without a deep understanding of the problem domain is nearly impossible. On the other hand mastering the disparate domains of expertise is a challenging and time consuming issue. For example in the absence of an ERP system the business analysis engineer or business analysis engineering group should take the followings under control:

- Currently used software applications in the company; there may be some heterogeneous applications that do not talk to each other

- Keeping track of customer orders from acceptance to fulfillment

- Managing the interdependencies of complex Bill of Materials and product structure

- Supply chain and interactions with suppliers and transportation infrastructure 
The above functionalities can be implemented in the following service groups:

- Object specifications

- Requirements and regulations

- ERP software features and asset specifications

Each of the above mentioned categories may be defined in terms of Ontologies. So at analysis time the business engineer could be aware of the consequences of the decisions made. The following scenario depicts the situation better.

Consider that a business process should be analyzed and mapped to a new ERP solution. The business engineer may explain the business process in terms of ontology and annotate it using some terms. In the next step the annotated object will be matched with an ERP process and also all pre conditions will be extracted from relevant regulation ontologies (safety regulations, material regulations ...) and checked. So by capturing the knowledge of problem domain the business engineer will be able to consider all requirements and the whole ERP implementation process will become more reliable.

\subsection{Use of Ontology-based Personal Information Management Systems}

Another challenge in Enterprise Information System is the detailed capture of activities and process information. ERP systems usually define a predefined sequence of activities which are in turn used to do process calculations. However, often the predefined concepts are not optimal and sometimes ambiguous in capturing the business activities. The question is how precise is the knowledge which is being captured automatically. Frequently, humans need to jump in, interpret the activities and modify the concerned process. A lot of knowledge still resides with experts, as tacit knowledge which has not been captured in a machine processable way due to lack of available technology. Now using ontologies, we are in a position to represent the expert's concepts in a formal way. The ambiguous processes can now be stated explicitly, and then can be read and processed by machines.

The time spent by a resource in a business process is crucial in calculating the overall product costs. The precise tracking of human resource time consumption is generally not possible. Consider the scenario where a marketing staff member might be talking on telephone to production staff for acquiring some useful information while preparing the tender documents for a new project, or customer support staff talking to him / her about an existing project. Apart from spending time in conversation, the production staff member starts a sequence of post-conversation activities to fulfill the query such as to consult the internet etc. Generally these intermittent requests from other departments are not tracked as far as the identification of the project and the time spent is concerned.

In our ontological personal information management system SemanticLIFE [14] we are capturing the information coming from different data sources. The different data sources are emails, process data running on your computer, your web browsing history, contacts in your address book, appointments in the calendar, chat sessions, 
file system monitor. Also we are in the process of implementing other data feeds like telephone log. The information is stored according to trivial ontology for each data source. In next step, domain ontology is made to interconnect these data sources according to the different types of user queries. Manual annotations and to some extent automatic associations are possible by using available metadata in incoming information items. For example it is possible to identify the project related with the web pages you visited for searching material about a technical query from your marketing counter part. The association is made based upon the following:

a. The Project ontology describing the project plan, resources, assignments, allocations, costs etc

b. The data feed ontology describing the individual data feeds, their attributes

c. Extraction of key words, and key concepts like time, location from the captured data feed

d. Mapping the instance of ontology from step b, onto entities from step a based upon key words from step c

A sample of our project ontology is as follows:

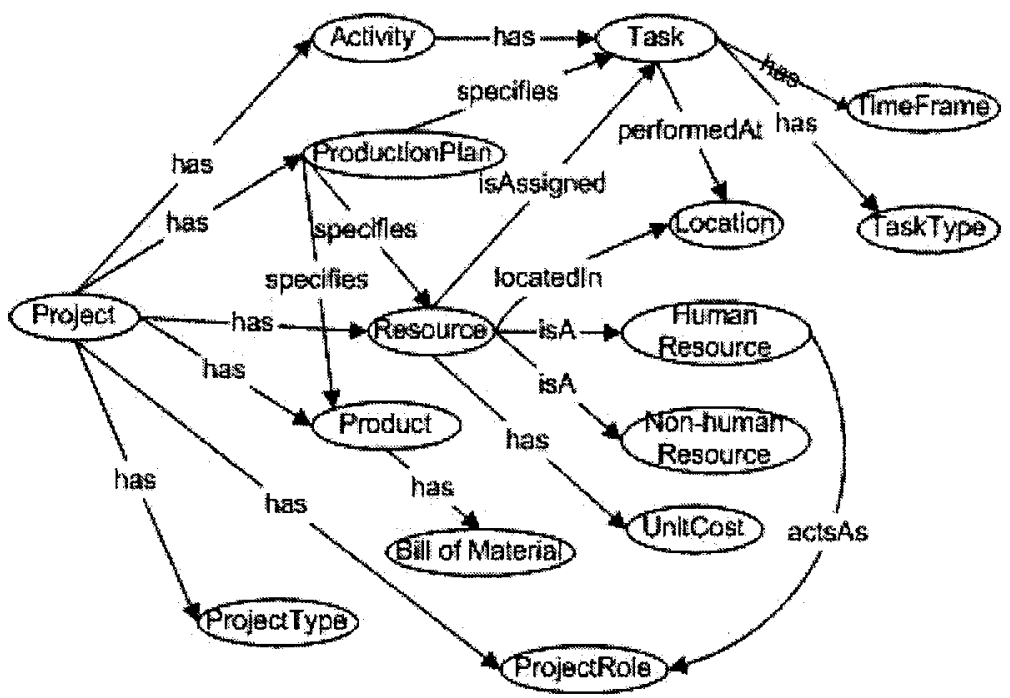

Fig. 1. Project Ontology

\subsection{Business Security}

As the closed domain of organization's applications opens to the outside world, there will be a growing need for enforcing complex policies whose satisfaction is not easy 
to manage via traditional security methods. By considering the fact that the average business loses three to six percent of annual revenue due to fraud, it goes without saying that the ERP security is of great importance. Enforcing context-sensitive policies in open domains requires the ability to opportunistically interleave policy reasoning with the dynamic identification, selection and access of relevant sources of contextual information. This requirement exceeds the capability of decentralized trust management infrastructures proposed so far, and calls for privacy and security enforcing mechanisms capable of operating external services [15].

On the highest level the security requirements of a typical business process can be broken down to "Task level security" and "Process level security" [16]. The task level security needs an "access security model" to define the constraints and access to system modules. On the other hand process security requires a "business security model" that may overlap with the access security model. Semantic Web paradigm may be used to address the complexity of our security models. As a first step, the structure of business (organization) may be captured in an ontology which essentially involves business actors. In the second step, the business processes and activities are modeled and expressed in another ontology schema.

To give a concrete example; an enterprise may have (among other activities) projects and processes. The first step is, to define ontology for these types of activities. For example, a project has a project manager, who is a person. A project consists of tasks that may have some subtasks. Tasks may be dependent and each task has attributes like start date, end date, man hours and so on. Each project must be authorized by a board of directors, the finance department and so on. The same has to be done for the process specification. A process has a process owner, has tasks, and so on.

Further more the actor's ontology should be defined that is essentially a map of the company's organizational chart plus description about customers, associates, business partners and may be system web services. As a matter of fact, all persons, organizational units or systems that have an active or passive role in a business activity must be taken into consideration.

After this the connection between the actor ontology and the organization/activities ontology can be formally described and this will be used to determine access control logic. For example one could define, that a person who is a director, has "read" access to all objects that are defined in the activity ontology except the ones that are private property of some other person [17].

\subsection{Sharing Business Processes}

By evolution of Service Oriented Architectures the outlook of the business is entirely changed. The processes and services are no more limited to an organization but they may be used internally by other systems and externally by business partners to automate and compose a business solution. Integration and process automation are two of the most important issues facing organizations today. By providing a standards-based approach to integration, the Web services and services-oriented 
architectures have provided significant help in addressing these challenges. However, Web Service standards apply at the syntax level of service interface specification and do not capture service semantics.

Web Services are the basic blocks of such collaboration environments, and an expert may compose useful scenarios by combining well-suited sets of Web Services. Still the person, who chooses the Web Services and chain them together, should be familiar with specific knowledge of domain services. Also, the expert should be familiar with Web Services at both syntax and semantic level. On the other hand as the number of available Web Services increases, it becomes more and more critical to have automated systems to help us identify the services that match specific requirements. Finding suitable Web Services depends on the metadata presented by Web Service providers including description of Web Services and their capabilities.

Ideally the Web Service descriptions should be machine interpretable to automate the service discovery and service composition scenarios in an unambiguous way. Adding Semantics to Web Services for presenting requirements and capabilities of Web Services is essential for achieving certainty and machine-interpretability. Semantics define the meaning of terms, such as the meaning of parameters or business objects and the meaning or intent of an operation. Semantics are often described in ontology as a formal and unambiguous definition of terms and their relationships. Typically, ontology includes a set of inference rules to allow reasoning on the concepts being defined.

Currently the Web Service Description Language (WSDL) [18] is used to syntactically describe the structure of a Web Service. Semantic Web Service standards leverage the capabilities of WSDL by providing a mechanism to annotate the capabilities and requirements of Web services with semantic concepts referenced from a semantic model. This mechanism will annotate the service and its inputs, outputs and operations. Additionally, it will be able to specify and annotate preconditions and effects of Web Services. These preconditions and effects together with the semantic annotations of inputs and outputs enable the automation of the service discovery process.

\section{Conclusions and Future Work}

The evolution of Semantic Web has opened a new window in IT and specially data engineering fields. During last few years most of Semantic Web related standards and specifications are elaborated and gradually it has attracted the attention of IT industry for developing enterprise solutions based on Semantic Web. ERP systems are a huge application area where the explicit use of semantics can make a significant difference in terms of company's productivity and increased revenues. The investigation is made as to how the semantic web technology can be useful in this domain. Also, the new challenges with its adoption in terms of security and privacy of enterprise information are explored. A solution is proposed using different ontologies for data sources, business processes, and security and privacy policies. 
The work is still under progress by enhancing our prototype SemanticLIFE towards collaborative environments and providing semantic feeds for enterprise systems.

\section{Acknowledgements}

This work is supported by the ASEAN-EU University Network Initiative, and the Higher Education Commission of Pakistan.

\section{References}

1. RFC 2396 - Uniform Resource Identifiers (URI): Generic Syntax, Berners-Lee T., Fielding R., Masinter L., IETF, August 1998, http://www.isi.edu/in-notes/rfc2396.txt. (February 28, 2006).

2. RDF/XML Syntax Specification (Revised) http://www.w3.org/TR/rdf-syntax-grammar/ (February 28, 2006).

3. RDF Semantics http://www.w3.org/TR/rdf-mt/ (February 28, 2006).

4. OWL Web Ontology Language Reference http://www.w3.org/TR/owl-ref/ (February 28, 2006).

5. T. Gruber, Toward Principles for the Design of Ontologies Used for Knowledge Sharing, International Journal of Human-Computer Studies 43(5-6), 907 (1995).

6. J. Handler, Agents and the Semantic Web, IEEE Intelligent Systems 16(2), 30-37, (2001).

7. Digital Enterprise Research Institute (DERI), http://www.deri.ie/ (February 28, 2006).

8. M. Sumner, Risk factors in enterprise-wide/ERP projects. Journal of Information Technology 15(4), 317-328, (2000).

9. R. Haugen and W.E. McCarthy, REA, A Semantic Model for Internet Supply Chain Collaboration, ACM Conference on Object-Oriented Programming, Systems, Languages, and Applications, 15-19 October 2000 Minneapolis, Minnesota USA.

10. The Supply-Chain Operations Reference-model (SCOR) http://www.supplychain.org/page.ww?\%20section=SCOR+Model\&name=SCOR+Model (February 28, 2006).

11. M. Fayez, L. Rabelo, and M. Mollaghasemi, Ontologies for Supply Chain Simulation Modeling, In the Proceedings of the 2005 Winter Simulation Conference, p. 2364-2370.

12. Ontology-Based Supply Chain Process Mapping (SCOR+) http://www.productivityapex.com/products/scor.asp (February 28, 2006).

13. J.M. Nicolau, On Thoughts About The Brain, in R. Josef Moreno-Diaz, J. Mira-Mira, ed., Brain Processes, Theories and Models, The MIT Press, p. 71-77, 1996.

14. M. Ahmed, H.H. Hoang, M.S. Karim, S. Khusro, M. Lanzenberger, K. Latif, E. Michlmayr, K. Mustofa, H.T. Nguyen, A. Rauber, A. Schatten., M.N. Tho, and A.M. Tjoa, 'SemanticLIFE' - A Framework for Managing Information of A Human Lifetime, Proceedings of the International Conference on Information Integration, Web-Applications and Services (IIWAS'04, 27-29 September 2004 Jakarta-Indonesia).

15. J. Rao and N. Sadeh, Semantic Web Framework and Meta-Control Model to Enforce Context-Sensitive Policies, Proceedings of the Semantic Web and Policy Workshop (4th International Semantic Web Conference, 7 November, 2005, Galway-Ireland). 
16. D. Huang, Semantic Policy-based Security Framework for Business Processes, In the Proceedings of the Semantic Web and Policy Workshop (4th International Semantic Web Conference, 7 November, 2005, Galway-Ireland).

17. E. Weippl, A. Schatten, S. Karim, and A. Tjoa, Semanticlife Collaboration: Security Requirements and Solutions - Security Aspects of Semantic Knowledge Management, In the Proceedings of 5 th International Conference on Practical Aspects of Knowledge Management, Vienna-Austria, LNAI/LNCS Springer-Verlag, 3336, p. 365-377, 2004.

18. Web Services Description Language http://www.w3.org/TR/wsdl (February 28, 2006). 